\title{
Idiopathic Crescentic Glomerulonephritis
}

National Cancer Institute

\section{Source}

National Cancer Institute. Idiopathic Crescentic Glomerulonephritis. NCI Thesaurus. Code C128143.

Crescentic glomerulonephritis, the cause of which is unknown. 